\title{
A hydrodynamical study of multiple-shell planetary nebulae
}

\section{Measuring the post-shock velocities in the shells ${ }^{\star}$}

\author{
R. L. M. Corradi ${ }^{1,2}$, M. Steffen ${ }^{3}$, D. Schönberner ${ }^{3}$, and R. Jacob ${ }^{3}$ \\ 1 Isaac Newton Group, Ap. de Correos 321, 38700 Santa Cruz de la Palma, Spain \\ e-mail: rcorradi@ing.iac.es \\ 2 Instituto de Astrofísica de Canarias, 38200 La Laguna, Tenerife, Spain \\ 3 Astrophysikalisches Institut Potsdam, 14482 Potsdam, Germany \\ e-mail: [msteffen; deschoenberner;rjacob]@aip.de
}

Received 13 February 2007 / Accepted 18 August 2007

ABSTRACT

\begin{abstract}
Context. Planetary Nebulae (PNe) display complex radial surface brightness profiles whose understanding is crucial for a correct interpretation of their formation and evolution. In particular, the intermediate shells commonly observed around the bright rims of PNe contain important information for the discussion of the mass loss rate at the end of the AGB, for the determination of the postAGB age of the central stars and the distance via the expansion parallax method, and for the analysis of line profiles for unresolved, faraway objects like extragalactic PNe.

Aims. This paper describes the dynamical properties of PNe shells and presents a new practical method to properly determine their expansion velocity.

Methods. The analysis is guided by realistic radiative-hydrodynamical simulations which allow to test and verify different methods for the kinematical study of PNe shells using high-resolution long-slit spectra.

Results. We show that the use of the derivative of the line profile allows us to determine accurately the post-shock gas velocity in the shells. This method is generally superior to other techniques previously used. In addition, if applied to long-slit data, it allows to determine the velocities of the shells even when they are very close to those of the PNe rims. Its application to 10 real PNe confirms the model predictions and previous conclusions about the substantial mass loss increase during the latest AGB evolution.

Conclusions. The method and discussion presented in this paper are meant to be used as a guide for a correct determination of some basic kinematic and evolutionary properties of Galactic and extragalactic PNe, including their expansion parallaxes.
\end{abstract}

Key words. planetary nebula: general

\section{Introduction}

Planetary nebulae (PNe) display multiple shells which are the result of a complex radiative-hydrodynamical evolution of the mass lost by the stellar progenitor at the end of the Asymptotic Giant Branch (AGB) and in the PN phase. In particular, during a significant fraction of their evolution round and elliptical PNe develop a triple-shell structure (cf. Perinotto et al. 2004; Schönberner et al. 2005b), consisting of:

- a bright inner rim, which is the thin shell behind the shock produced by the fast post-AGB wind hitting the slow AGB wind. The density structure and expansion of the rim are driven by the pressure of the thermalized fast stellar wind inside it (the "hot bubble");

- an intermediate, thick shell, accelerated by the shock caused by the expansion of the ionization front through the neutral AGB wind, and whose structure is governed by the thermal pressure maintained by the heating from photoionization;

- an outer, large and much fainter AGB halo.

From the observational point of view, owing to its brightness the rim is the most widely studied structure of PNe in terms of its

* Based on observations obtained at the $3.5 \mathrm{~m}$ NTT and CAT telescopes of the European Southern Observatory in Chile, and at the $2.6 \mathrm{~m}$ NOT telescope operated by NOTSA in the Spanish Observatorio del Roque de Los Muchachos of the Instituto de Astrofísica de Canarias. physical, chemical, and kinematic properties (see e.g. Corradi et al. 2000b, Paper I). However, valuable information is also contained in the shell. Theories predict that the shell expansion velocity is mainly determined by the slope of the circumstellar density profile at the end of the AGB (Perinotto et al. 2004; Schönberner et al. 2005b). A combined observational study of the surface brightness profiles of haloes and of the expansion velocity of shells can confirm these predictions and provide quantitative information on the substantial increase in mass loss rate that is expected at the very end of the AGB. In addition, the shell outer radius and expansion speed give a better estimate of the kinematic post-AGB age of PNe than the corresponding quantities for the rim (Schönberner et al. 2005a). Moreover, it has been recently shown (Perinotto et al. 2004; Mellema 2004; Schönberner et al. 2005a) that it is generally not correct to couple angular expansion measurements with Doppler-shift velocities in order to determine the distance of PNe (the so-called expansion parallax method). This is because different kind of motions - pattern and matter motions for the expansion in the plane of the sky and along the line-of-sight, respectively - are considered. Even so, the correction factors to be applied in order to get right distances using the expansion parallax are significantly smaller for the shells than for the rims.

The determination of the expansion velocities of real PN shells, however, is not an easy task, as shells are generally faint, their emission along certain line-of-sights is overwhelmed by 
the rim emission, and the strong velocity gradients through the (thick) shells give rise to complex line profiles. A detailed study of the expansion properties of PN rims and shells was previously presented in Schönberner et al. (2005b); there, the shell velocities were computed from the emission line profiles along the line-of-sight through the centre of the nebulae. This methods fails, however, in those cases where the difference in velocities between rim and shell is small or even negative. In this paper, therefore, we aim at further improving these determinations by taking advantage of the whole information contained in long-slit spectra, obtaining an accurate estimation of the gas post-shock velocity in the shells. This method works also in cases where the velocities of rim and shell remain unresolved in the central line profile.

In Sect. 2, we present synthetic long-slit spectra computed from radiative-hydrodynamical simulations. The best measure of the post-shock gas velocity in the shells is found to be the fit, as a function of distance from the centre, of the peaks of the line profile first derivative. In Sect. 3, the analysis is applied to real objects, and compared with our previous results and with other studies in the literature. Discussion and conclusions are presented in Sect. 4. Note that the analysis in this paper will be limited to the [N II] and [O III] collisionally excited emission lines only. They were chosen because they are usually among the brightest lines in the rims and shells of PNe and have much lower thermal broadening ( $\simeq 5 \mathrm{~km} \mathrm{~s}^{-1}$ at $\left.T_{\mathrm{e}} \simeq 10000 \mathrm{~K}\right)$ than the similarly bright $\mathrm{H} \alpha$ hydrogen line $\left(22 \mathrm{~km} \mathrm{~s}^{-1}\right)$, allowing to resolve fainter velocity components in the line profiles.

\section{Synthetic spectra}

\subsection{The hydrodynamical PN models}

The basic characteristics of the hydrodynamical PN models used in the present work for the calculation of synthetic line profiles have been described elsewhere (e.g. Perinotto et al. 2004, and references therein), so it is sufficient to give only a brief summary of the main points here. Starting from a given initial spherical density distribution representing the stellar wind envelope produced by the previous mass loss on the tip of the AGB, we model the dynamical evolution of the circumstellar gas under the influence of the fast wind and the UV radiation field of the evolving post-AGB central star. The employed radiation hydrodynamics code assumes spherical symmetry, but otherwise includes all the relevant physics necessary to model the formation and evolution of PNe. A robust numerical method to handle compressible flows with strong shocks, and a detailed treatment of timedependent photo-ionization for up to 9 elements (79 ions) are among the essential features of this code.

Models A-E listed in Table 1 constitute a time sequence taken from a standard hydrodynamical PN sequence with a central star (CS) of mass $M=0.595 M_{\odot}$. As described in Schönberner et al. (2005a), the stellar track was combined with a realistic initial circumstellar wind envelope, obtained from a two-component hydrodynamical simulation of a dust-driven AGB outflow (see Steffen et al. 1998), based on stellar evolution on the upper AGB with mass loss according to Blöcker (1995). The assumed AGB mass loss rate results in a final mass of $M=0.605 M_{\odot}$, slightly higher than the assumed mass of $M=0.595 M_{\odot}$ defining the post-AGB evolution of the PN central star. However, this minor inconsistency is of no concern in the present context. The structure of the initial model is shown in Schönberner et al. (2005a, Fig. 2). In that work it was demonstrated that this kind of initial condition leads to realistic
Table 1. Hydrodynamical PN models used to compute synthetic longslit spectra (see text). Columns show: (1) Model name; (2): central star effective temperature; (3) central star luminosity; (4) post-AGB age; (5) and (6): inner radius of rim, $r 0$, and outer radius of rim $=$ inner radius of shell, $r 1$, relative to the outer radius of the shell, $r 2$; (7)-(9) flow expansion velocities at $r 0, r 1$, and $r 2$.

\begin{tabular}{c|rrr|cc|ccc}
\hline \hline Model & $\begin{array}{r}T_{\text {eff }} \\
{[\mathrm{kK}]}\end{array}$ & $\begin{array}{r}L_{\mathrm{CPN}} \\
{\left[L_{\odot}\right]}\end{array}$ & $\begin{array}{r}\text { Age } \\
{[\mathrm{yr}]}\end{array}$ & \multicolumn{2}{|c|}{$r 0$} & \multicolumn{2}{|c|}{$r 1$} & \multicolumn{2}{|c}{$v 0$} & \multicolumn{2}{c}{$v 2$} \\
& {$[\mathrm{r} 2]$} & \multicolumn{2}{|c}{$\left[\mathrm{km} \mathrm{s}^{-1}\right]$} \\
$(1)$ & $(2)$ & $(3)$ & $(4)$ & $(5)$ & $(6)$ & $(7)$ & $(8)$ & $(9)$ \\
\hline A & 66.3 & 5238 & 5317 & 0.30 & 0.50 & $\mathbf{1 1}$ & $\mathbf{8}$ & $\mathbf{2 8}$ \\
B & 77.9 & 5099 & 5986 & 0.31 & 0.50 & $\mathbf{1 3}$ & $\mathbf{1 0}$ & $\mathbf{2 9}$ \\
C & 88.3 & 4893 & 6530 & 0.32 & 0.51 & $\mathbf{1 6}$ & $\mathbf{1 2}$ & $\mathbf{3 0}$ \\
D & 120.8 & 3937 & 8057 & 0.37 & 0.55 & $\mathbf{2 2}$ & $\mathbf{1 6}$ & $\mathbf{3 3}$ \\
E & 141.3 & 2733 & 9197 & 0.40 & 0.59 & $\mathbf{2 6}$ & $\mathbf{1 9}$ & $\mathbf{3 6}$ \\
F & 97.7 & 4669 & 6994 & 0.44 & 0.57 & $\mathbf{2 1}$ & $\mathbf{1 4}$ & $\mathbf{2 9}$ \\
\hline
\end{tabular}
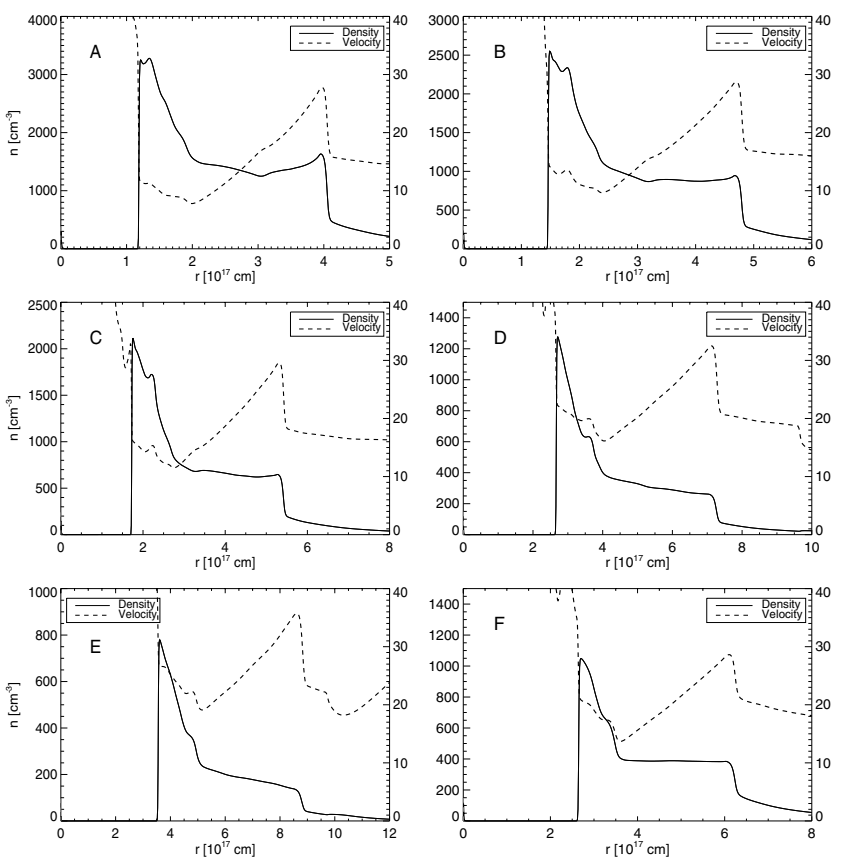

Fig. 1. Radial profiles of density (atomic nuclei per $\mathrm{cm}^{3}$ ) (solid line, left ordinate) and expansion velocity $\left(\mathrm{km} \mathrm{s}^{-1}\right)$ (dotted line, right ordinate) for the models listed in Table 1.

hydrodynamical PN models with typical double-shell (plus halo) structures whose geometry and surface brightness distribution is in close agreement with that of real PNe (Schönberner et al. 2005a, Fig. 7).

Model $\mathrm{F}$ was taken from another hydrodynamical PN sequence which is based on the same central star track $\left(M=0.595 M_{\odot}\right)$, but assumes a somewhat different AGB mass loss history with a smaller maximum rate, resulting in a less massive initial AGB wind envelope. The lower density of the circumstellar envelope leads to a higher expansion velocity of the rim, compared to models $\mathrm{A}-\mathrm{E}$, while the expansion rate of the outer shell is rather similar. Hence, the difference between the expansion velocity of shell and rim is smaller in model F (cf. Table 1), which was the reason for selecting this model.

The radial density structure and the corresponding expansion velocity is shown in Fig. 1 for all models (A-F). We note that the shell is characterized by a constant positive velocity gradient, while the density is roughly constant or slightly decreasing with radius. In all cases, the shell expands faster than the rim (cf. Table 1). 

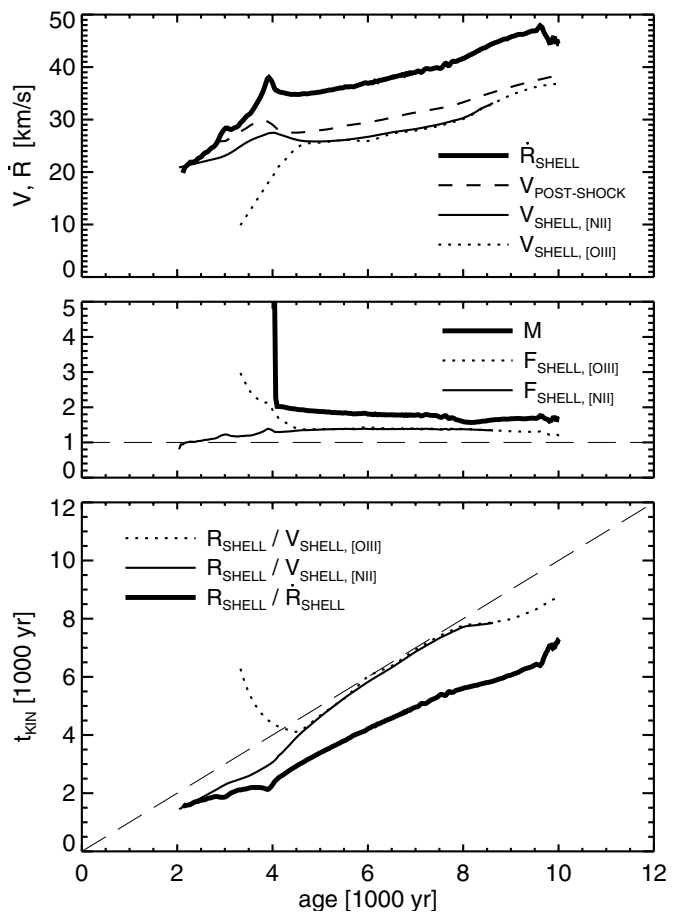

Fig. 2. Evolution with post-AGB age of the kinematic properties of the shell of a PN model with a $0.595 M_{\odot}$ CS. Top: velocities of the shell's shock, $\dot{R}_{\text {shell }}$ and of the flow immediately behind the shock, $V_{\text {post-shock }}$. The velocities $V_{\text {shell }}$ as derived from the Gaussian decomposition of the synthetic central line profiles of $[\mathrm{N} \mathrm{II}]$ and $[\mathrm{O} \mathrm{III}]$ are also displayed. Middle: mach number of the shell's shock, $M=\left(\dot{R}_{\text {shell }}-V_{\text {pre-shock }}\right) / C$, with $C$ being the isothermal sound speed ahead of the shock, and the ratios $F=\dot{R}_{\text {shell }} / V_{\text {shell }}$ of the shock speed and the gas velocities as derived from Doppler split emission lines. Bottom: kinematic ages, $t_{\text {kin }}$, determined from the various combinations of shock position and velocities.

\subsection{Shell velocities}

The kinematics of a PN shell is more closely illustrated in Fig. 2. The figure is identical with the left panel of Fig. 4 in Schönberner et al. (2005a). In the figure, material velocities are indicated by $V$, motions of structures - like shocks - by $\dot{R}$, with $R$ denoting their radial position. We see in the figure that the shell velocities $V_{\text {shell }}$, derived from the peak value of Gaussians fitted to the profiles of the [O III] or [N II] lines, are always smaller than the post-shock velocities $V_{\text {post-shock }}$ of the gas (top panel). This is a consequence of the radial velocity gradient within the shell. Note that in all panels the [N II] and [O III] lines behave differently for ages less than $4500 \mathrm{yr}$ because of the ionization stratification while the PN model is optically thick in the Lyman continuum.

Fortunately, the factor $F$ to correct for the difference between the Doppler velocities and the shock speed $\dot{R}_{\text {shell }}$ is rather small $(\simeq 1.5)$ and even close to unity during the optically thick phase for the [N II] lines (middle panel, $\$ 4500 \mathrm{yr}$ ). The lower Doppler speeds, however, make partly up for the shell's acceleration when used for the estimate of kinematical ages, $R_{\text {shell }} / V_{\text {shell }}$, such that they appear to be a reasonable approximation to the real post-AGB ages (bottom panel). Again, this statement is only true if the $[\mathrm{N}$ II] lines are used during the optically thick phase.

\subsection{Long-slit simulations}

Since the hydrodynamical PN models described above provide all the necessary physical information (like expansion velocity, particle densities, electron temperature, and line emissivity as a function of radius), they can be used to compute synthetic emission line profiles. For the central line-of-sight, synthetic line profiles have been compared with corresponding observations of real PNe by Schönberner et al. (2005b), who found a good agreement between model and observation, both qualitatively and quantitatively. In particular, the models reproduce the faint "shoulder" seen in many line profiles, which is the signature of the fast, lower density shell superimposed on the main component due to the slow, higher density rim.

We have developed a tool for computing synthetic line profiles from the hydrodynamical models for arbitrary impact parameters and slit geometries, based on the code by Gesicki et al. (1996). Since the nebula is optically thin in all lines, the task is just to add up the emissivity along a given line-of-sight for each frequency, properly taking into account the differential Doppler shift due to the radial expansion. We have used this tool here to simulate long-slit spectra for direct comparison with observed spectra, including an appropriate instrumental line broadening (Gaussian with $F W H M=6 \mathrm{~km} \mathrm{~s}^{-1}$ ).

Figure 3 shows some illustrative velocity profiles in the [O III] $500.7 \mathrm{~nm}$ emission line for models $\mathrm{C}$ and D for the lineof-sight through the centre of the nebula (top-left), in a intermediate position in the rim (top-right), and at various slit positions at distances $d$ from the centre outside the projected outer radius of the rim out to the edge of the shell. A qualitatively similar behavior applies to the other models and other slit positions. It is evident that, given the large velocity gradient through the shells, their thickness, and the projection effects, line profiles are complex and can be hardly fit, except close to the centre $(d \simeq 0)$, by analytical functions or multi-Gaussian fit, especially in real nebulae where asymmetries and inhomogeneities make profiles more irregular (see Sect. 3).

In Schönberner et al. (2005b), we have computed the shell velocity by fitting four Gaussians (two for the rim, and two for the shell) to the central profile $(d=0)$ of real PNe. We have applied this method to all models, and results are listed in Col. 2 of Table 2. In the last two columns of the Table, the post-shock gas velocity $V_{\text {post-shock }}$ of the models and the total range of velocities through the shell (generally in the form of a monotonic increase with radius up to $V_{\text {post-shock }}$, see Fig. 1) are also listed (cf. Table 1). As illustrated also in the bottom panel of Fig. 7, the 4-Gaussian fit (filled squares) generally underestimates $V_{\text {post-shock }}$ by some $20 \%$, the agreement being slightly better for models at late evolutionary stages. It is also obvious that if the shell velocity is not substantially larger than the rim velocity, this method is not applicable, as the high velocity shoulders of the shell are lost in the wings of the dominant rim emission. To measure the shell velocity in the latter case, one can take advantage of the information available in long-slit spectra at lineof-sights offset from the nebular centre. For spherical symmetry, such a spectrum at any position angle provides all the information required.

As shown in Appendix A by means of a simplified analytical model, using the first derivative of the long-slit line profiles with respect to Doppler velocity to find the steepest slope in the line wings is a reliable method to determine the maximum expansion velocity $\left(V_{\text {post-shock }}\right)$ in the shells of the PNe. With the aim to determine the post-shock velocity, we have computed the first derivative of the synthetic long-slit line profiles for the PN models listed in Table 1 at all slit positions. In the derivative spectra, the first peak (and the last negative one, given the symmetrical, receding part of the nebula) correspond to the maximum expansion velocity in the shell, projected along the line-of-sight 

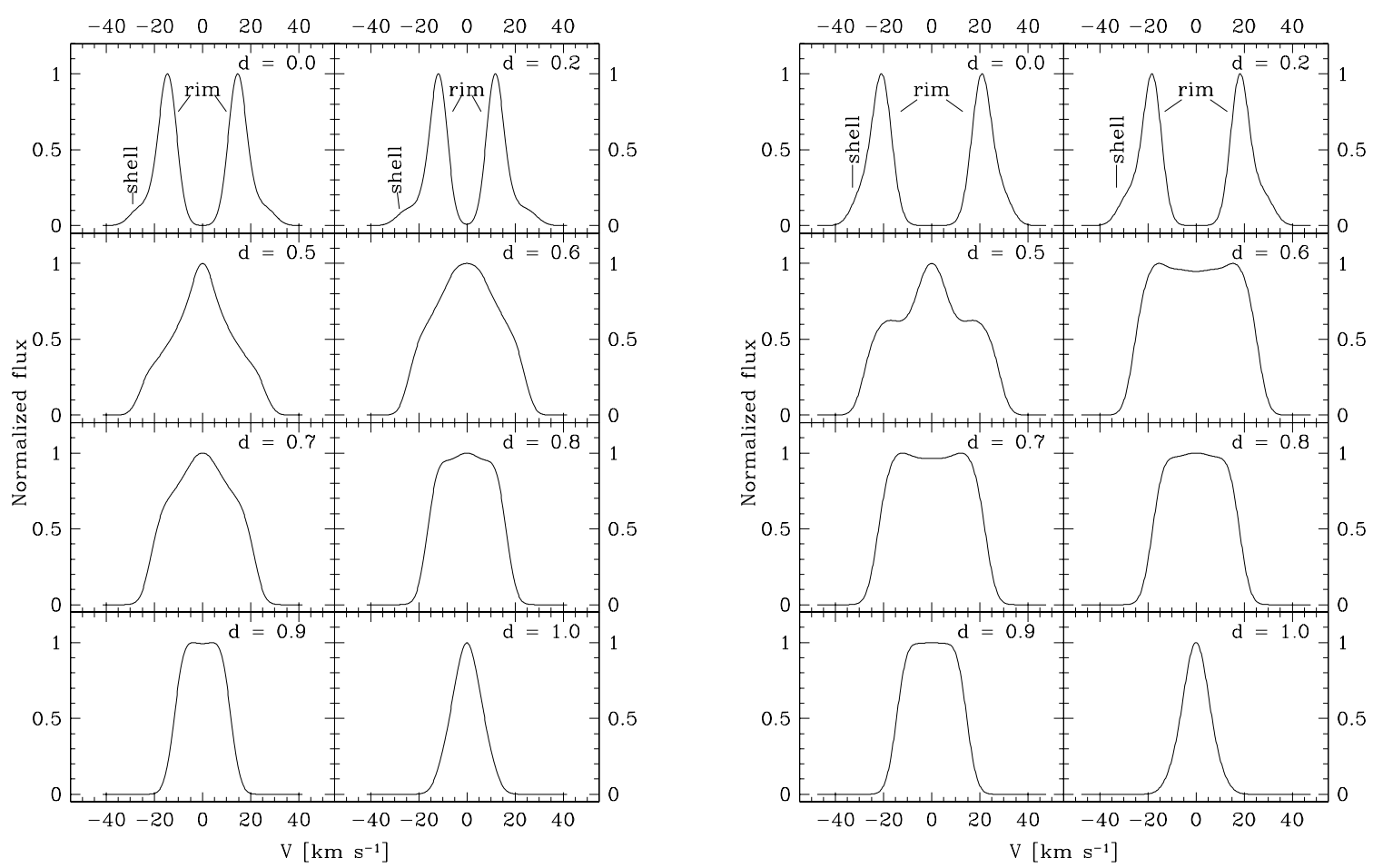

Fig. 3. [O III] velocity profiles for models C (left) and D (right), at different projected distance $d$ from the central star (normalized to the shell's radius). In the top panels the velocity profile is dominated by the rim emission; the signature of the shell are the faint high velocity "shoulders", which are clearly seen in model C but are only marginally visible in the more evolved model D. The other panels show the evolution of line profiles from just outside the projected outer radius of the rim out to the edge of the shell.

Table 2. Shell velocities (in $\mathrm{km} \mathrm{s}^{-1}$ ) measured by different methods; fitting 4 Gaussians to the central profile (Col. 2), fitting a spherical model to the FWHM of the profiles at distances from the centre larger than the outer radius of the rim (Col. 3); fitting the spherical model to the full-width at 10\% intensity (Col. 4, ":" means that these figures are uncertain); and fitting the peaks of the line profile first derivative (Col. 5, see text and Fig. 6). In Cols. 6 and 7, the post-shock gas velocity and the total velocity range across the shell, respectively, are indicated.

\begin{tabular}{ccccc|cr}
\hline \hline Model & $\begin{array}{c}\text { 4 Gaussians } \\
\text { at centre } \\
(1)\end{array}$ & $\begin{array}{c}\text { Fit } F W H M \\
\text { long-slit } \\
(3)\end{array}$ & $\begin{array}{c}\text { Fit } F W 10 \% \\
\text { long-slit } \\
(4)\end{array}$ & $\begin{array}{c}\text { Fit peaks of line } \\
\text { profile derivative } \\
(5)\end{array}$ & $\begin{array}{c}\text { Post-shock } \\
\text { gas velocity } \\
(6)\end{array}$ & $\begin{array}{r}\text { Total } \\
\text { range } \\
(7)\end{array}$ \\
\hline A & 21 & 26 & $33:$ & 29 & $\mathbf{2 8}$ & $\mathbf{8 - 2 8}$ \\
B & 23 & 26 & $34:$ & 29 & $\mathbf{2 9}$ & $\mathbf{1 0 - 2 9}$ \\
C & 24 & 25 & $34:$ & 31 & $\mathbf{3 0}$ & $\mathbf{1 2 - 3 0}$ \\
D & 25 & 31 & $38:$ & 32 & $\mathbf{3 2}$ & $\mathbf{1 6 - 3 2}$ \\
E & 29 & 32 & $40:$ & 35 & $\mathbf{3 5}$ & $\mathbf{1 9 - 3 5}$ \\
F & 25 & 28 & $34:$ & 29 & $\mathbf{2 9}$ & $\mathbf{1 4 - 2 9}$ \\
\hline
\end{tabular}

at that slit position (see Appendix A). Two illustrative examples selected from the profiles in Fig. 3 are shown in Fig. 4.

Figure 5 is essentially the same as Fig. 2 but with the shell velocities measured from the derivative of the central profiles instead. We see that this method allows a direct determination of the post-shock velocity with only a negligible error ${ }^{1}$. The correction to the real expansion speed during the optically thin phase is smaller than for the case shown in Fig. 2, $F$ being around $1.2 \ldots 1.3$ (middle panel). Again, the expansion velocity based on the derivative provides also a reasonable estimate of the kinematical expansion age (bottom panel), although it is slightly more underestimated than in the case of Fig. 2.

Coming back to the long-slit simulations, for each model the velocities of the outer peaks of the line profile derivative are displayed in Fig. 6 as empty circles as a function of distance $d$. The shell expansion velocity was then computed by fitting these position-velocity plots by means of a spherical thin shell model

\footnotetext{
${ }^{1}$ During the optically thick phase, [O III] should not be used (Fig. 5)!
}

with given size (the outer radius of the shell) and expansion velocity $V$. The fits are shown as solid lines in Fig. 6. It is clear that the peaks of the line profile derivative are extremely well fitted by the spherical shell model, providing an excellent measure of $V_{\text {post-shock }}$ (see Col. 5 of Table 2, and the large empty circles in Fig. 7). Note that in the earlier models the method works even in the central regions where the rim contribution to the line emission is dominant (but is confined to lower velocities than those that characterize the outer layers of the shell). Only when the rim velocity approaches $V_{\text {post-shock, namely when the difference }}$ between both velocities becomes smaller than about $10 \mathrm{~km} \mathrm{~s}^{-1}$ (models D, E and F), the signature of the shell is not recognizable any longer in the central regions. Even in these cases, however, the fit using a large range of slit positions out to the edge of the shell correctly provides $V_{\text {post-shock}}$.

Similar fits have been attempted using observables that are easier to measure, especially in spectra of real PNe where asymmetries and noise are present. We have therefore measured the full width at half maximum $(F W H M)$ of line profiles at all slit 


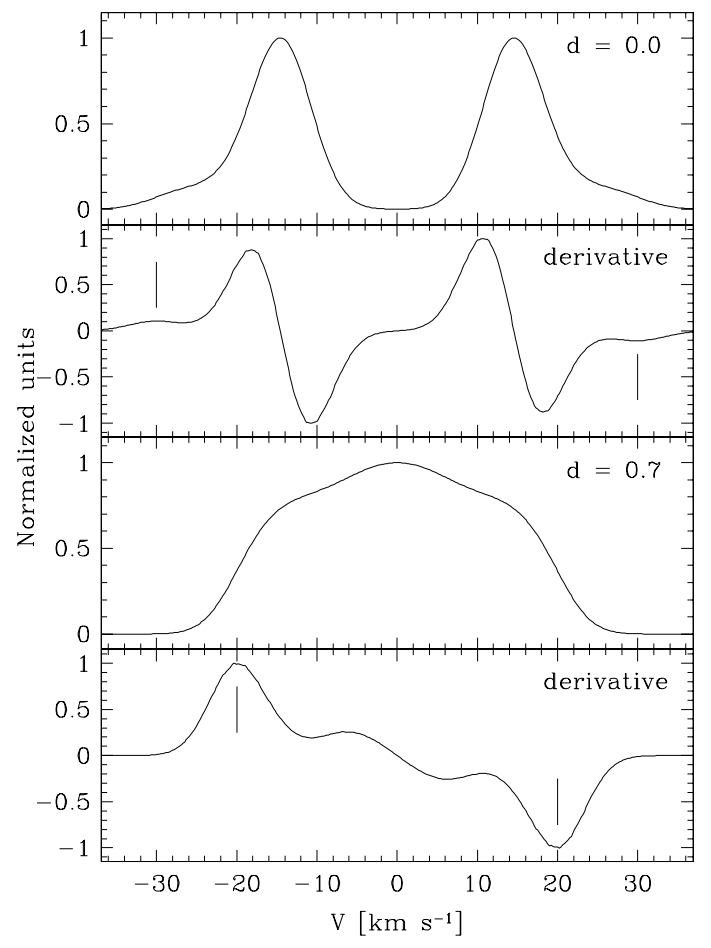

Fig. 4. Line profiles and their derivatives for the central profile of model $\mathrm{C}$ and in the shell at $d=0.7$ (cf. Fig. 3). Vertical lines show the velocities measured at the outer peaks of the line profile derivative (see text).

positions, as well as the full-width at $10 \%$ intensity of the emission peak $(F W 10 \%)$. The $F W H M$ velocities are plotted as small filled circles in Fig. 6, which shows how they are much more sensitive to the rim contribution in the inner regions than the peaks of the derivative spectra. In spite of that, if only the outer regions are considered, the fit of the $F W H M$ velocities provides a reasonable measure of $V_{\text {post-shock }}$, although it systematically underestimates it by $\leq 20 \%$ (Col. 3 of Table 2, and empty squares in Fig. 7). On the contrary, the fit to the FW10\% with a spherical shell model is generally poor, providing uncertain estimates which systematically overestimates $V_{\text {post-shock }}$ by 15 to $20 \%$ (Col. 4 of Table 2, and small black circles in Fig. 7).

We have also attempted to make a multi-Gaussian fit to the line profiles at all slit positions: in order to have a good fit, a large and variable number of Gaussians (up to 7 in some models and slit positions) is needed, and only the outermost component, which is usually the faintest one, provides a fair (albeit systematically underestimated) measure of $V_{\text {post-shock }}$. This makes this method arbitrary and thus unreliable, and in particular hardly applicable to real spectra which have more asymmetrical profiles and limited $\mathrm{S} / \mathrm{N}$ ratios. For this reason, we do not further discuss this method in the following.

Summarising, our conclusion from the analysis of the synthetic long-slit spectra is the following:

- The fit of the velocities corresponding to the outer peaks of the line profile derivative, as a function of distance from the centre in long slit spectra, gives an accurate estimate of the post-shock gas velocity in PN shells. Only in the cases where the rim velocity approaches that of the shell, the fit should be limited to the outer regions where the rim contribution is less important or absent.

- The fit of the FWHM of line profiles, an observable which can be measured more easily than the peaks in the spectrum

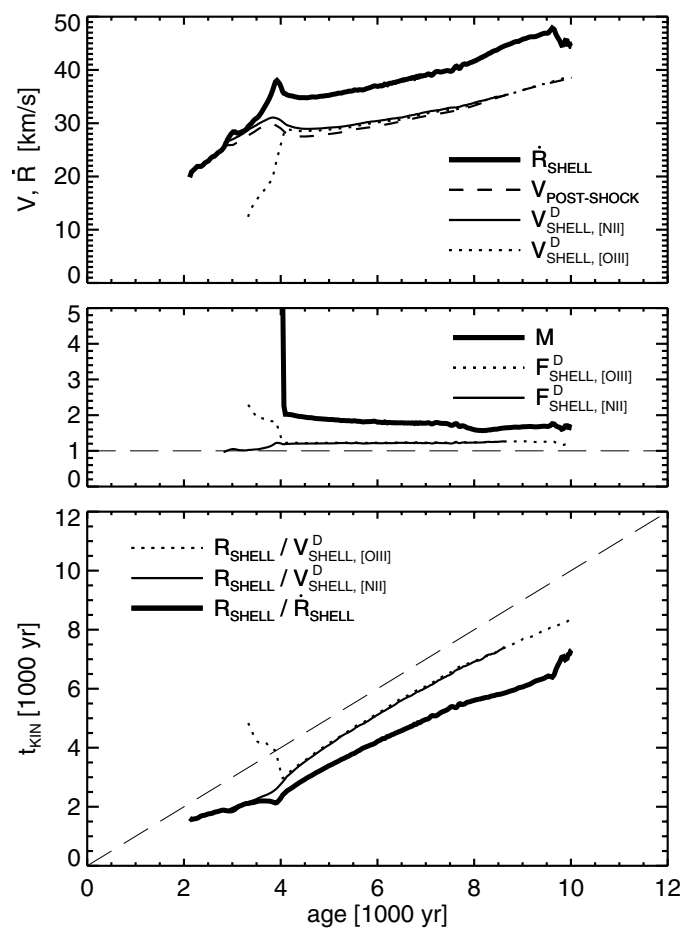

Fig. 5. Same as in Fig. 2, but with the Doppler velocities of the shell of the central profile replaced by the velocities measured at the outermost peaks of the line profile derivative, $V^{\mathrm{D}}$ (see top panels of Fig. 4).

derivative in real spectra with relatively low $\mathrm{S} / \mathrm{N}$ ratio, also provides a fair measure of $V_{\text {post-shock }}$ although underestimating it by up to $20 \%$. But note that the $F W H M$ taken only for the central profile $(d=0)$ overestimates the rim expansion and severely underestimates, by up to $50 \%$ (model A), the shell expansion speed (cf. Fig. 6).

- A 4-Gaussian fit of the line profile through the centre of the nebula significantly underestimates $V_{\text {post-shock }}$ (by 15 to $25 \%$, cf. Table 2).

\section{Application to real PNe}

The method based on the line profile derivative was applied to a sample of PNe with circular or mildly elliptical morphologies in the attempt to improve the determination of their shell velocities and to derive $V_{\text {post-shock. The long-slit echelle spectra }}$ from the ESO $3.5 \mathrm{~m} \mathrm{NTT}$ and $2.6 \mathrm{~m}$ NOT telescopes presented in Schönberner et al. (2005b) were reanalysed. Details on the instrumental configuration are given in that paper. The objects analysed are listed in Table 3, and greyscale plots of their [O III] long-slit spectra are shown in Fig. 8. From that figure, it is clear that line profiles in the shells are generally more complex and irregular than in the models (cf. the two plots at the bottom of the figure). In some cases, this is due to the presence of inhomogeneities, the most obvious case being NGC 7662 that possesses remarkable small-scale structures in its shell (Balick et al. 1998). In spite of these inhomogeneities, the variation of line profiles along the slit for real nebulae follow the same pattern as in the models; this is further illustrated by the comparison of line profiles for NGC 3242 and NGC 1535 in Fig. 9 with the model spectra in Fig. 3. For an easier comparison, distances from the centre for the profiles have been selected to be roughly the same in the two figures. 


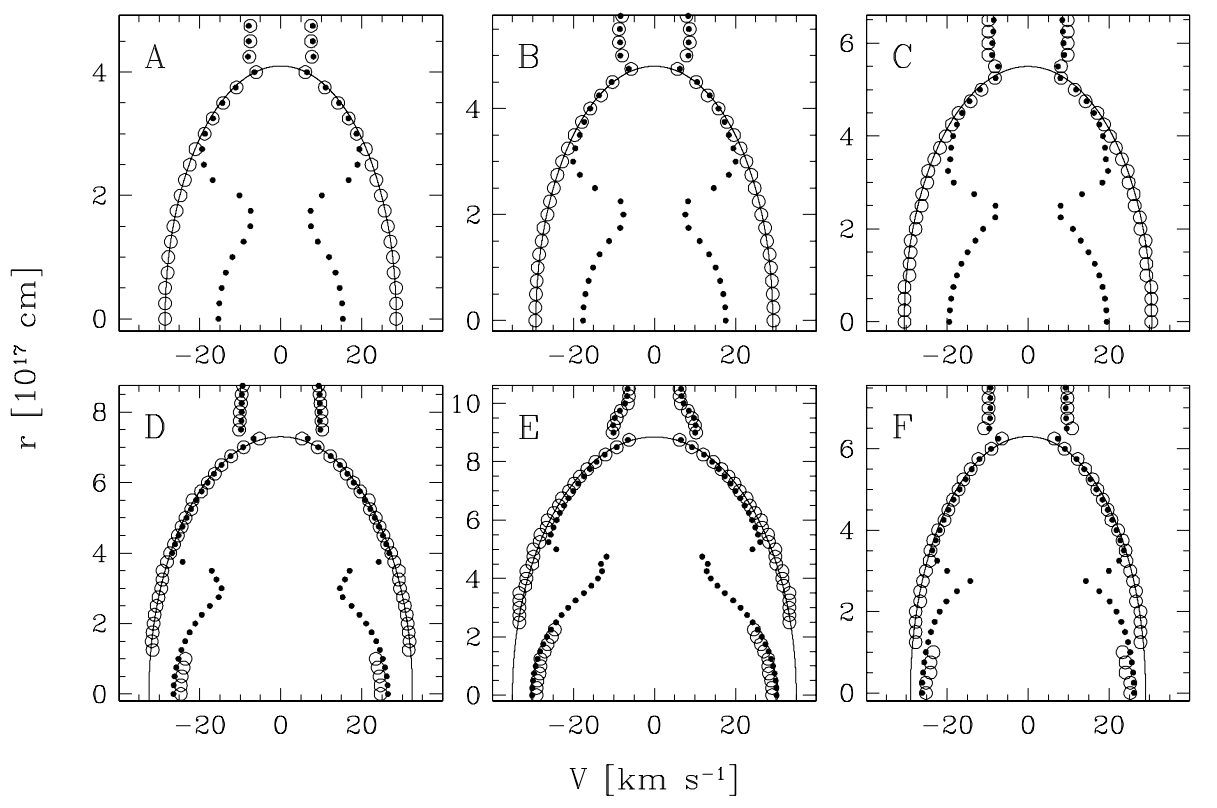

Fig. 6. Solid lines are the fit to the velocities measured at the peaks of the line profile derivative (open circles) at distances along the synthetic long-slit spectra large enough that the rim emission does not dominate. Black smaller circles indicate the velocity measured at the FWHM of line profiles. Outside the region of the fit ellipses emission comes from the faint AGB halo.

Table 3. Shell velocities (in $\mathrm{km} \mathrm{s}^{-1}$ and for the [O III] line) determined by the fit of the velocities corresponding to the outer peak of the line profile derivative as a function of distance from the centre along the slit (Col. 3), from the 4-Gaussian fit of the central profile (Col. 4), and from other sources in the literature (Col. 5, reference in Col. 6). The [O III] expansion velocity of the rim is indicated in Col. 7. The CAT data are only for $[\mathrm{N}$ II]

\begin{tabular}{|c|c|c|c|c|c|c|}
\hline \multicolumn{7}{|c|}{ NTT long-slit data } \\
\hline Object & $\begin{array}{c}\text { Slit } \\
\text { PA }\end{array}$ & $\begin{array}{c}V_{\text {shell }} \\
\text { peak derivative }\end{array}$ & $\begin{array}{c}V_{\text {shell }}^{\dagger} \\
\text { 4 Gaussians at centre }\end{array}$ & $\begin{array}{c}V_{\text {shell }} \\
\text { (other) }\end{array}$ & $\begin{array}{l}\text { Reference } \\
\text { (other) }\end{array}$ & $V_{\text {rim }}^{\dagger}$ \\
\hline IC 2448 & -44 & 35 & 33 & & & 18 \\
\hline IC 2448 & +46 & 35 & 33 & & & 17 \\
\hline Мy 60 & -50 & $30-45$ & 40: & & & 23 \\
\hline NGC 1535 & +20 & 32 & * & 27 & Multi-Gaussian fit, Schönberner et al. (2005b) & 22 \\
\hline NGC 2610 & 0 & 42 & $*$ & 46: & Lehmann \& Hildebrandt, priv. comm. & 34 \\
\hline NGC 3242 & +60 & 40 & 36 & 33 & Lehmann \& Hildebrandt, priv. comm. & 19 \\
\hline \multirow[t]{2}{*}{ NGC 7662} & +48 & 32 (equat.) & $*$ & 50 & Guerrero et al. (2004) & 26 \\
\hline & & 42 (polar) & $*$ & 60 & Guerrero et al. (2004) & 26 \\
\hline NGC 7662 & +138 & 30 (equat.) & $*$ & 50 & Guerrero et al. (2004) & 26 \\
\hline \multicolumn{7}{|c|}{ CAT short-slit data } \\
\hline Object & $\begin{array}{l}\text { Slit } \\
\text { PA }\end{array}$ & $\begin{array}{c}V_{\text {shell }} \\
\text { peak derivative at centre }\end{array}$ & $\begin{array}{c}V_{\text {shell }}^{\dagger} \\
4 \text { Gaussians at centre }\end{array}$ & $\begin{array}{c}V_{\text {shell }} \\
\text { (other) }\end{array}$ & $\begin{array}{l}\text { Reference } \\
\text { (other) }\end{array}$ & $V_{\text {rim }}^{\dagger}$ \\
\hline M 1-46 & & 32 & 26 & 26 & Guerrero et al. (1996) & 12 \\
\hline NGC 5882 & & 48: & 40 & $>38$ & Corradi et al. (2000a) & 22 \\
\hline NGC 6629 & & 34 & 27 & & & 6 \\
\hline Tc 1 & & 30 & 26 & & & 12 \\
\hline
\end{tabular}

${ }^{\dagger}$ From Schönberner et al. (2005b). : Quoted as uncertain in Schönberner et al. (2005b). * $V_{\text {shell }}$ could not be measured because rim and shell have similar velocities.

As done for the models, we have computed line profile derivatives and measured the velocities of the outermost peaks at all slit positions from the centre to the outer edge of the shells. The FWHM of line profiles at slit positions between the outer radii of the rims and the outer radii of the shells were also measured. Data were binned along the spatial direction by an amount that depends on the seeing conditions and the $\mathrm{S} / \mathrm{N}$ ratio of the spectra, ranging from 0!'7 for NGC 7662 to 1'.3 3 for NGC 2610. The plots of the velocities from the derivative spectra (empty circles), as well as from the FWHM of lines (filled smaller circles) are presented in Fig. 10. Following the conclusions of Sect. 2, the shell's post-shock velocity was then computed by fitting a spherical shell model to the velocities of the peaks of the line profile derivative (solid lines in the figure). Only for NGC 7662 at $\mathrm{PA}=+48^{\circ}$, given the tilted appearance of its position-velocity plot, an ellipsoidal prolate model whose major axis is inclined by $50^{\circ}$ with respect to the line-of-sight (as in Guerrero et al. 2004), and with a polar velocity of $40 \mathrm{~km} \mathrm{~s}^{-1}$ and an equatorial one of $32 \mathrm{~km} \mathrm{~s}^{-1}$, was fitted. The corresponding shell velocities for all PNe are listed in Col. 3 of Table 3.

For My 60, one side of the nebula indicates a lower shell velocity than the opposite side. Both values are indicated in Fig. 10 and Table 3.

In NGC 1535 and NGC 2610, the rim velocity comes close to $V_{\text {post-shock }}$, and as found in the models at late evolutionary stages the determination of the shell's velocity in the innermost regions might be disturbed by the rim emission. This is why the measured velocity drops in the first few arcseconds from the centre, which were not taken into consideration for the fit. 


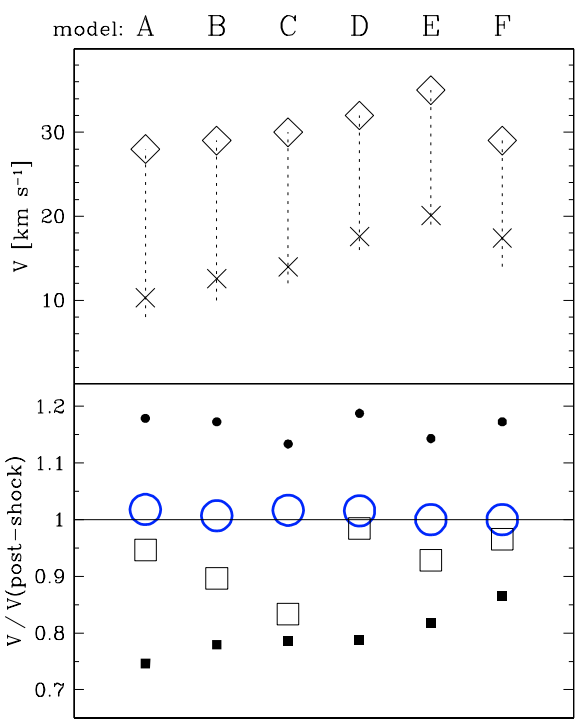

Fig. 7. Top: the post-shock gas velocity $V_{\text {post-shock }}$ (open diamonds), the total velocity range through the shell (dashed lines), and the rim expansion speed as measured from the split of the [O III] emission in the central spectrum (crosses). Bottom: the expansion velocity of the shell, relative to $V_{\text {post-shock }}$, as determined from the different methods discussed in the text: large empty circles (blue in the electronic version of the paper) are the result of the fit of the peaks of the line profile derivatives along the slit (Fig. 6); large empty squares the fit of the FWHM of line profiles along the slit; small filled circles the fit of the FW10\%; and filled squares the 4-Gaussian fit of the central profile.

In NGC 7662, the velocity variation with distance is particularly irregular because of the structured density distribution in the shell and rim and their ellipsoidal geometry tilted on the lineof-sight (cf. Guerrero et al. 2004). In spite of this, an useful range of shell velocities could be determined also for this nebula.

In NGC 3242, a sudden drop in the measured velocities occurs right outside the projected outer radius of the rim. We have no explanation for this effect, as the high-velocity signature of the shell in the innermost regions is clearly visible at all slit positions, and given its significantly larger velocity it is not disturbed by the rim emission.

Note also that the fit of the $F W H M$ velocities would lead to lower estimates of $V_{\text {post-shock }}$, in the sense predicted by the discussion in Sect. 2, but to a somewhat larger extent than expected by the application of the method to the models. On the other hand, the 4-Gaussian fit of the central profiles, while giving systematically lower figures than the analysis of the line profile derivative, provides a better agreement than suggested in Sect. 2 .

This work improves the determination of the post-shock gas velocity in the shells of these PNe. New measurements are provided for NGC 1535, NGC 2610 and NGC 7662, where the difference between $V_{\text {post-shock }}$ and the rim velocity is smaller than $10 \mathrm{~km} \mathrm{~s}^{-1}$, and where therefore the shell's signature in the central velocity profiles is partially or completely hidden inside the much stronger rim emission. Finally, our shell velocities for NGC 7662 are a factor $\geq 1.5$ smaller than the value quoted by Guerrero et al. (2004), which was determined by fitting a position-velocity ellipse enclosing all the [O III] emission in their echellograms. It thus appears that the method of Guerrero et al. (2004) significantly overestimates the shell expansion velocity.

Additionally, we also reanalysed the "short-slit" CAT data presented in Schönberner et al. (2005b, Fig. 9 and Table 2 therein). These $[\mathrm{N} \mathrm{II}]$ profiles are limited to the central part of

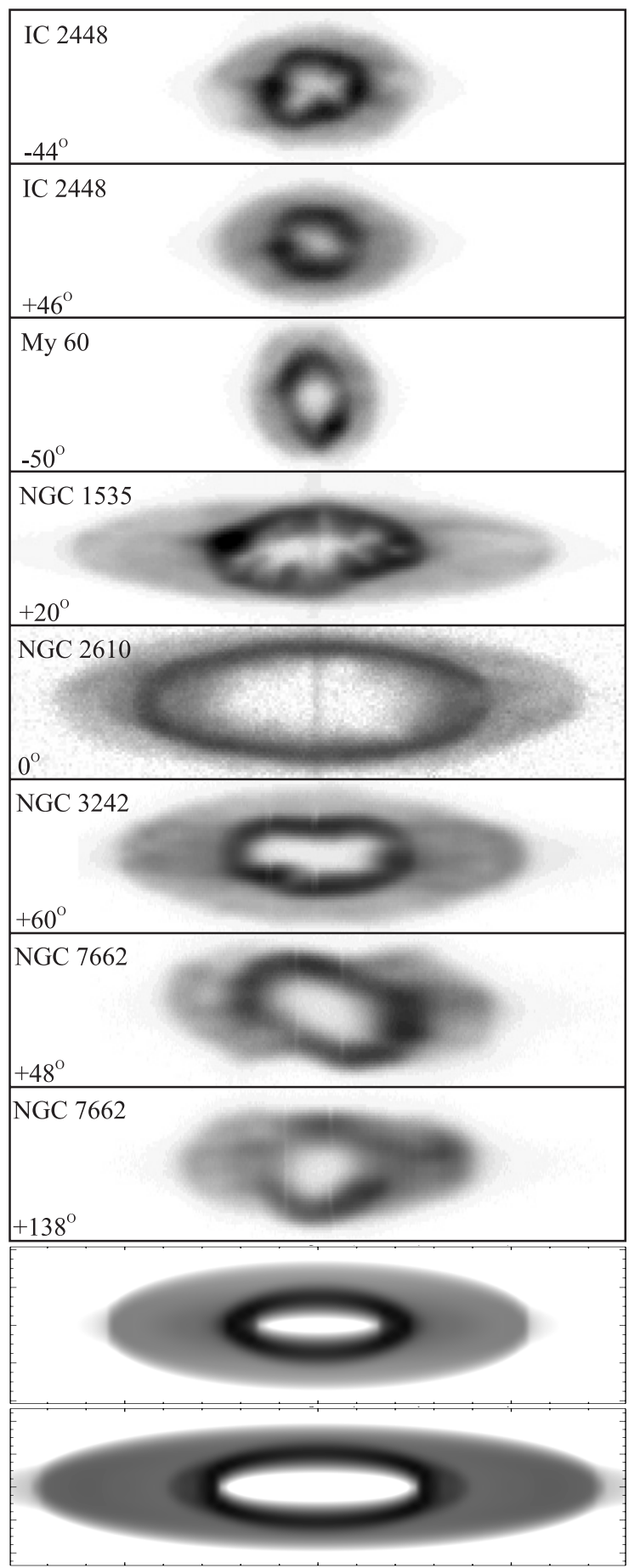

Fig. 8. Greyscale logarithmic plots of the [O III] line of the target $\mathrm{PNe}$ in the echelle long-slit spectra. Each box is $60^{\prime \prime}$ along the horizontal direction, and $100 \mathrm{~km} \mathrm{~s}^{-1}$ along the vertical one. The dark "ellipses" in the inner region of each PN correspond to the rim's emission, while the paler, outer emission is the contribution of the shells. At the bottom, similar plots are shown for models C (second last row) and D (last row).

the PNe, but the signature of the shell is clearly apparent in all four cases. Thus the derivative of the line profile is expected to provide an improved measure of the shell's post-shock velocity for all objects, as suggested by the models (cf. Fig. 5, upper panel). The results are also listed in Table 3, and two examples are shown in Fig. 11.

A final remark is given on the effect of noise on the determination of line profile derivatives. The method seems to be robust 

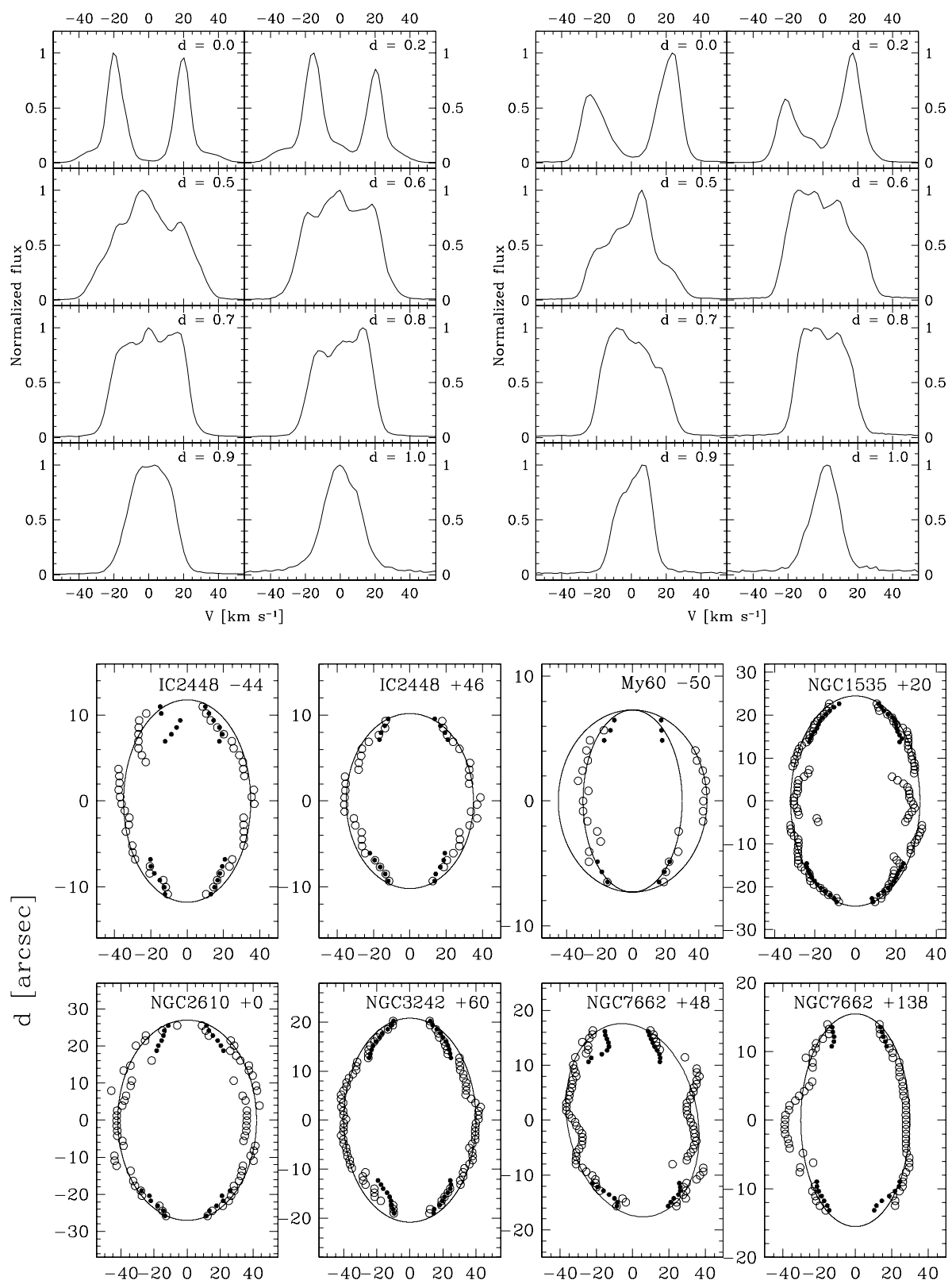

$\mathrm{V}\left[\mathrm{km} \mathrm{s}^{-1}\right]$
Fig. 9. [O III] velocity profiles NGC 3242 (left) and NGC 1535 (right) at various distances $d$ (normalized to the shell's outer radius) from the centre along $\mathrm{PA}=+60^{\circ}$ and $\mathrm{PA}=+20^{\circ}$, respectively. $d$ has been selected so as to roughly correspond to the distances in Fig. 3 for the model profiles.

Fig. 10. Velocities along the slit measured at the peaks of the line profile derivative (empty circles) and at the FWHM of the profiles (filled circles) for the sample of PNe with NTT longslit data. The fits with expansion velocities as listed in Table 3 are indicated by the solid lines.

against noise for data of the quality as presented in this article. This can be seen in Fig. 11, which is illustrative of the level of noise typical of all our spectra. It is also supported by the consistency of the measurements within each nebula (the empty circles in Fig. 10): the derivatives, which are computed in a complete independent way at each slit position, show a regular, relatively smooth trend with distance in spite of noise, inhomogeneities and asymmetries in the real nebulae.

\section{Discussion and conclusions}

Guided by realistic hydrodynamic models of $\mathrm{PNe}$, we have shown that the use of the derivative of the line profile allows to determine a value from the shell's velocity field which is virtually equal to the post-shock velocity. This methods is superior to other methods used previously because (i) the correction to the real expansion rate, i.e. that of the shell's shock, is quite small, and because (ii) it works also reliably in cases where rim and shell velocities are similar and cannot be separated by using central line profiles (provided that long-slit observations covering the whole object are at hand). Our new method could be very useful if one wants to combine angular expansion rates (of the shell!) with the measured and corrected real expansion velocity in order to estimate distances to individual objects (expansion parallaxes).

By means of long-slit spectra we were able to determine the shell expansion of 3 objects (NGC 1535, NGC 2610, NGC 7662) for which a direct determination from the central profile is not possible. The velocities range from about 30 to about $40 \mathrm{~km} \mathrm{~s}^{-1}$ and do not differ from those of the other objects where shell and rim expansion can be measured from the central profile.

As already demonstrated in Schönberner et al. (2005b), such large expansion velocities are only possible if the shell expands into an environment whose radial density profile, if approximated by a power law, falls off as $\rho \propto r^{-3 \ldots-3.3}$. Indeed, in Monreal-Ibero et al. (2005) we reported that the halo density 

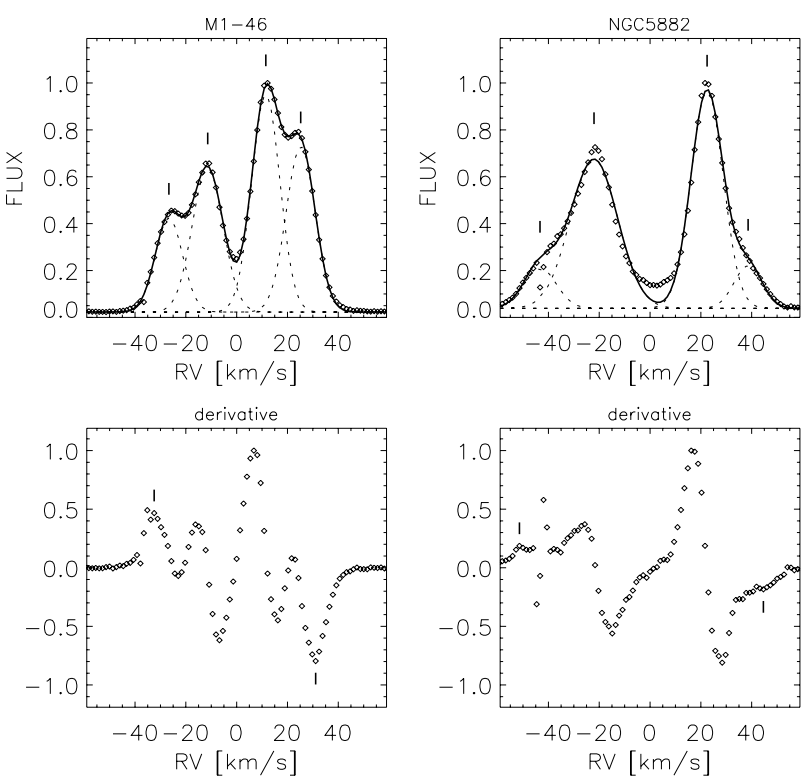

Fig. 11. High-resolution central line profiles of [N II] $658.3 \mathrm{~nm}$ and their derivatives for M 1-46 and NGC 5882. Top: 4 Gaussian decompositions (dashed) and their fit to the line profiles (cont. line). Rim and shell velocities are defined by the positions of the individual Gaussians. Bottom: derivatives of the line profiles, with the outermost peaks (or dips) marked (cf. Fig. 4).

of NGC 3242 falls radially off approximately with $r^{-2.8}$, in good agreement with what one would expect for the expansion speed of the shell of $40 \mathrm{~km} \mathrm{~s}^{-1}$.

We also would like to emphasize again (see Schönberner et al. 2005b) that the expansion velocities of the PNe analysed here appear to be larger than the "canonical" value of $20 \mathrm{~km} \mathrm{~s}^{-1}$ quoted in the literature and used often in statistical analyses. The solution of this discrepancy lies in the fact that most velocity measurements used the line split of the strong components which are the signature of the bright rim. The average expansion velocity of the rim of the 10 objects listed in Table 3 is indeed close to $20 \mathrm{~km} \mathrm{~s}^{-1}$ ! The analysis of spatially integrated profiles will yield a similar result because the bright rim will dominate the line profile as well. The problem of the correct expansion velocities is especially important for extragalactic applications. From our hydrodynamic modelling we expect that the expansion behavior of PNe depends also strongly on the metallicity of their environment (see Schönberner et al. 2005c, for details). Velocities based on FWHM measurements of integrated profiles are certainly not very helpful to tackle the problem since all the important information from the rather faint shells may get lost. For studying PNe in extragalactic systems, we urgently need a better method for the determination of expansion velocities.

Acknowledgements. R.L.M.C. acknowledges funding from the Spanish AYA2002-0883 grant.

\section{Appendix A: A parameter study with a semi-analytical shell model}

In order to illustrate how the surface brightness distribution and the shape of the line profile emitted by the shell depend on the expansion velocity law and distribution of line emissivity, we have performed a parameter study using a simple spherical PN shell model. The model is characterized by the following
Table A.1. Semi-analytical PN shell models used to compute synthetic surface brightness distributions and long-slit spectral line profiles. The columns list the following parameters. (1) Model designation; (2) ratio of inner to outer shell radius; (3) expansion velocity at outer edge of shell; (4) slope of velocity profile (Eq. (A.2)); (5) slope of emissivity profile (Eq. (A.3)); (6) electron temperature of shell; (7) atomic weight of emitting ion; (8) full width at half maximum of the Gaussian instrumental profile.

\begin{tabular}{c|cccc|ccc}
\hline \hline Model & $r_{\mathrm{i}}$ & $\begin{array}{c}v_{\mathrm{s}} \\
{\left[\mathrm{km} \mathrm{s}^{-1}\right]} \\
(1)\end{array}$ & $\alpha$ & $\beta$ & $\begin{array}{c}T_{\mathrm{e}} \\
{[\mathrm{K}]}\end{array}$ & $w$ & $\begin{array}{c}F W H M \\
{\left[\mathrm{~km} \mathrm{~s}^{-1}\right]}\end{array}$ \\
T1 & 0.5 & $\mathbf{3 0}$ & $(4)$ & $(5)$ & $(6)$ & $(7)$ & $(8)$ \\
\hline T2 & 0.5 & $\mathbf{3 0}$ & $\mathbf{2 . 0}$ & $\mathbf{1 . 0}$ & 8000 & 16 & 6.0 \\
$\mathrm{~T} 3$ & 0.5 & $\mathbf{3 0}$ & $\mathbf{0 . 0}$ & $\mathbf{1 . 0}$ & 8000 & 16 & 6.0 \\
T4 & 0.5 & $\mathbf{3 0}$ & $\mathbf{1 . 0}$ & $\mathbf{0 . 5}$ & 8000 & 16 & 6.0 \\
T5 & 0.5 & $\mathbf{3 0}$ & $\mathbf{2 . 0}$ & $\mathbf{0 . 5}$ & 8000 & 16 & 6.0 \\
T6 & 0.5 & $\mathbf{3 0}$ & $\mathbf{0 . 0}$ & $\mathbf{0 . 5}$ & 8000 & 16 & 6.0 \\
\hline T7 & 0.5 & $\mathbf{3 0}$ & $\mathbf{1 . 0}$ & $\mathbf{2 . 0}$ & 8000 & 16 & 6.0 \\
T8 & 0.5 & $\mathbf{3 0}$ & $\mathbf{2 . 0}$ & $\mathbf{2 . 0}$ & 8000 & 16 & 6.0 \\
T9 & 0.5 & $\mathbf{3 0}$ & $\mathbf{0 . 0}$ & $\mathbf{2 . 0}$ & 8000 & 16 & 6.0 \\
\hline
\end{tabular}

parameters. The geometry is fixed by $r_{i}$, the ratio of inner and outer shell radius:

$r_{\mathrm{i}}=R_{\mathrm{i}} / R_{\mathrm{o}}$.

The expansion velocity across the shell is assumed to obey a linear law of the form

$v(r)=v_{\mathrm{s}}\{1-\alpha(1-r)\}, \quad r_{\mathrm{i}} \leq r \leq 1$,

where $v_{\mathrm{s}}$ is the expansion velocity at the outer edge of the shell, corresponding to the post-shock velocity. The case $\alpha=1$ corresponds to a Hubble flow, $v(r)=v_{\mathrm{s}} r$ (see Fig. A.1a). The line emissivity per unit volume is also parametrized by a linear relation as

$\eta(r)=1-(1-\beta) \frac{1-r}{1-r_{\mathrm{i}}}, \quad r_{\mathrm{i}} \leq r \leq 1$,

where $\beta=\eta\left(r_{\mathrm{i}}\right) / \eta\left(r_{\mathrm{o}}\right)$ specifies the ratio of the emissivities at inner and outer shell radius. $\beta=1$ gives a constant emissivity (see Fig. A.1b). We also need to specify the electron temperature $T_{\mathrm{e}}$ of the shell and the atomic weight $w$ of the line emitters for the calculation of the isotropic thermal line broadening. Both $T_{\mathrm{e}}(=8000 \mathrm{~K})$ and $w(=16$ to represent oxygen $)$ are constant throughout the entire shell. Finally, we emulate the instrumental line broadening by a Gaussian profile with a full width at half maximum of $F W H M=6 \mathrm{~km} \mathrm{~s}^{-1}$, corresponding to a spectral resolving power of 50000 . Table A.1 lists the parameter combinations of the computed test models.

For each of the models listed in Table A. 1 we have computed the resulting surface brightness distribution, $S(d)$, and the emission line profiles $I(v, d)$ where $r_{\mathrm{i}} \leq d \leq 1$ is the impact parameter, and $v$ is the Doppler velocity. These observable quantities are obtained by numerical integration of the (Doppler-shifted) emissivity along the line-of-sight. The code used for this purpose is completely independent of the tool used to compute synthetic line-profiles from the hydrodynamical PN models as described in Sect. 2.3.

Figure A.1 illustrates the dependence of the surface brightness profile $S(d)$ on the slope $\beta$ of the emissivity across the shell. As expected, the gradient of $S(d)$ becomes steeper as $\beta$ is increased. Observed surface brightness distributions suggest that $\beta>1$. 

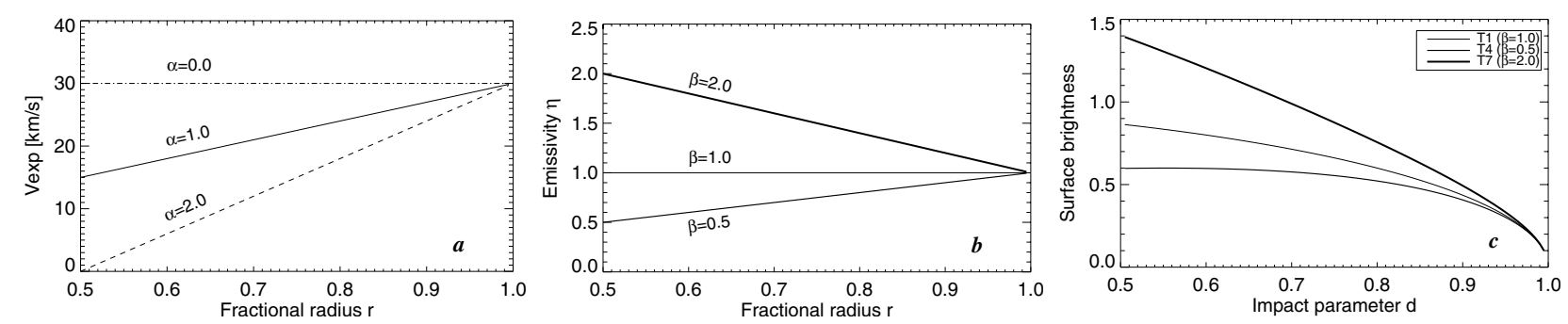

Fig. A.1. a) Radial profile of the shell expansion velocity for the three test cases $\alpha=1.0,2.0,0.0$. b) Radial profile of the shell emissivity for the three test cases $\beta=1.0,0.5,2.0$. c) Comparison of the surface brightness distributions computed for test models T1 $(\beta=1.0)$, T4 ( $\beta=0.5)$, and $\mathrm{T} 7(\beta=2.0)$.
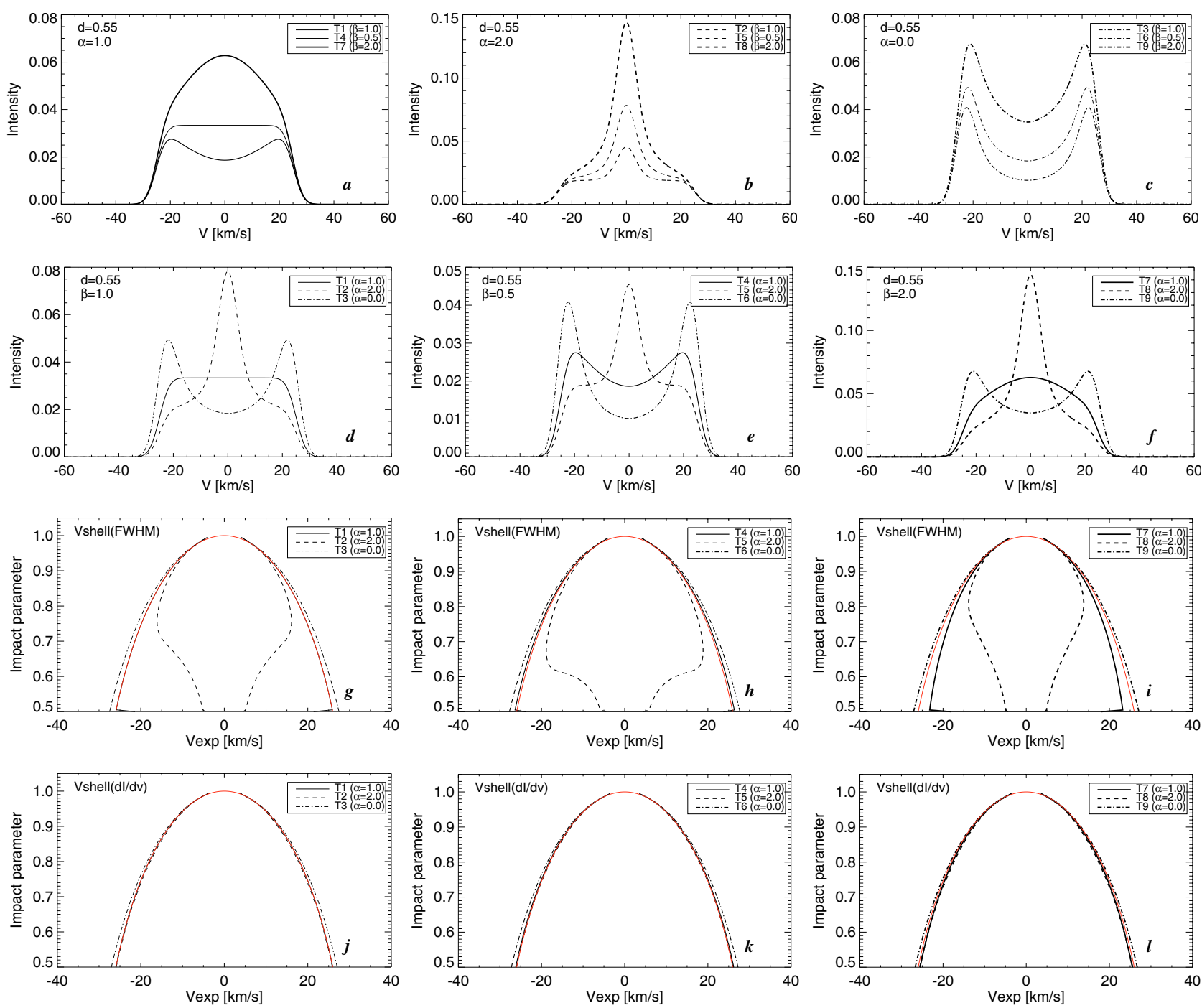

Fig. A.2. Line profiles computed from test models T1 .. T7 at impact parameter $d=0.55$. At this impact parameter the (projected) expansion velocity is $25.1 \mathrm{~km} \mathrm{~s}^{-1}$. First row: dependence of the line shape on the emissivity gradient $\beta$ at fixed velocity structure (panel a)): $\alpha=1.0$, b): $\alpha=2.0, \mathbf{c}): \alpha=0.0$ ). Second row: dependence of the line shape on the velocity gradient $\alpha$ at fixed emissivity structure (panel d)): $\beta=1.0$, e): $\beta=0.5, \mathbf{f}$ ): $\beta=2.0$ ). Third row (panels $\mathbf{g}$ ), h), i)): velocity ellipse resulting from the $F W H M$-method for all synthetic line profiles with $d \geq 0.5$. Last row (panels $\mathbf{j}), \mathbf{k}), \mathbf{l}$ )): velocity ellipse resulting from the derivative method for all synthetic line profiles with $d \geq 0.5$.

The resulting line profiles are displayed in Fig. A.2. Each panel of the first row shows the dependence of the line shape on $\beta$ at fixed velocity structure $(\alpha)$. The line profiles are rearranged in the panels of the second row to demonstrate the dependence of the line shape on $\alpha$ at fixed emissivity law $(\beta)$.

The line profile is particularly simple in the case of $\mathrm{T} 1$, where $\alpha=1, \beta=1$. It can be shown analytically that, in the absence of any intrinsic and instrumental line broadening, the profile is of box shape, extending from $-v_{\mathrm{d}}$ to $+v_{\mathrm{d}}$, where $v_{\mathrm{d}}=v_{\mathrm{s}} \sqrt{1-d^{2}}$ is the projected shell velocity at impact parameter $d$ (here $26 \mathrm{~km} \mathrm{~s}^{-1}$ at $d=0.5$ ). Hence, the width of the profile corresponds exactly to the expansion velocity at the outer edge of the shell. In the presence of intrinsic and instrumental line broadening, the line profile is the convolution of the box-shaped 
profile with the combined intrinsic and instrumental profile. If the latter can be represented by a Gaussian, $g(v) \sim \exp \left\{-v^{2} / w^{2}\right\}$, the total line profile can be written in terms of the error-function,

$p(v, d) \sim \operatorname{erf}\left\{\left(v+v_{\mathrm{d}}\right) / w\right\}+\operatorname{erf}\left\{\left(-v+v_{\mathrm{d}}\right) / w\right\}$.

The full width at half maximum of this profile is $F W H M=$ $2 v_{\mathrm{d}}=2 v_{\mathrm{s}} \sqrt{1-d^{2}}$, i.e. the measurement of FWHM gives the correct shell velocity.

The first derivative of $p(v, d)$ with respect to $v$ yields

$p^{\prime}(v, d) \sim g\left(x+v_{\mathrm{d}}\right)-g\left(x-v_{\mathrm{d}}\right)$.

The extrema of $p^{\prime}(v, d)$ are located at $\pm v_{\mathrm{d}}$, giving the true projected maximum shell velocity.

For the above example, the $F W H M$-method and the derivative method both give the correct result $\left(26 \mathrm{~km} \mathrm{~s}^{-1}\right.$ at $\left.d=0.5\right)$ as seen in Fig. A.2 (panels $g$ and $j$ ). If the emissivity increases with radius $(\beta=0.5)$, the profile is no longer box-shaped but has a central depression. But also in this case, both methods work very well (panels $h$ and $k$ ). If the emissivity decreases with radius $(\beta=2.0)$, the profile shows a central peak. In this case, the $F W H M$-method significantly underestimates the shell velocity (panel $i$ ), while the derivative method works well (panel $l$ ).

If the velocity gradient is steeper than $\alpha=1$ (T2, T5, T8), the profiles show a strong central peak and pronounced shoulders (see Fig. A.2, panel b). In this case, the FWHM-method fails completely (see dashed line in panels $g, h, i$ ). In contrast, the derivative method gives almost perfect results (see dashed line in panels $j, k, l$ ).

If the velocity is constant across the shell, $\alpha=0$ (T3, T6, T9), the line profiles show a central depression and intensity maxima at high Doppler velocity (see Fig. A.2, panel c). In this situation, the $F W H M$-method slightly overestimates the shell velocity (see dot-dashed line in panels $g, h, i$ ). The derivative method also tends to overestimate the shell velocity, but the results are better than for the FWHM-method (see dot-dashed line in panels $j, k, l)$. However, the error is quite small for both methods.
Of the computed test models, T7 seems to come closest to observed profiles (cf. Fig. 9), indicating that real PNe have $\alpha \gtrsim 1.0, \beta \gtrsim 1.0$. The somewhat more complex fine structure of the observed profiles can be reproduced by modified models where the emissivity law is a non-monotonic function of radius (e.g. emissivity minimum inside the shell). Such details depend sensitively on the density and ionization structure of the shell.

Our parameter study strongly suggests that the derivative method is more reliable than the FWHM-method. For model T7, for example, the derivative method gives somewhat higher shell expansion velocities than the $F W H M$-method. However, the line profiles of real PNe can be more complex, and the derivative method might be impossible to apply if the $\mathrm{S} / \mathrm{N}$ ratio is too low.

The analysis of the central line profile $(d=0)$ is more involved since both the shell and the rim properties enter. The construction of simple analytical models then requires at least 2 more more free parameters. The exploration of this extended parameter space is beyond the scope of the present study.

\section{References}

Balick, B., Alexander, J., Hajian, A. R., et al. 1998, AJ, 116, 360

Blöcker, T. 1995, A\&A, 297, 727

Corradi, R. L. M., Gonçalves, D. R., Villaver, E., Mampaso, A., \& Perinotto, M. 2000a, ApJ, 542, 861

Corradi, R. L. M., Schönberner, D., Steffen, M., \& Perinotto, M. 2000b, A\&A, 354,1071

Gesicki, K., Acker, A., \& Szczerba, R. 1996, A\&A, 309, 907

Guerrero, M. A., Manchado, A., Stanghellini, L., \& Herrero, A. 1996, ApJ, 464, 847

Guerrero, M. A., Jaxon, E. G., \& Chu, Y.-H. 2004, AJ, 128, 1705

Mellema, G. 2004, A\&A, 416, 623

Monreal-Ibero, A., Roth, M. M., Schönberner, D., Steffen, M., \& Böhm, P. 2005, ApJ, 628, L139

Perinotto, M., Schönberner, D., Steffen, M., \& Calonaci, C. 2004, A\&A, 414, 993

Schönberner, D., Jacob, R., \& Steffen, M. 2005a, A\&A, 441, 573

Schönberner, D., Jacob, R., Steffen, M., et al. 2005b, A\&A, 431, 963

Schönberner, D., Jacob, R., Steffen, M., \& Roth, M. M. 2005c, in Planetary Nebulae as Astronomical Tools, ed. R. Szczerba, G. Stasinska, \& S. K. Gorny, AIP Conf. Proc., 804, 269

Steffen, M., Szczerba, R., \& Schönberner, D. 1998, A\&A, 337, 149 